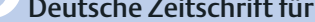 Osteopathie}

Offizielles Organ folgender Verbände

Verband der Osteopathen Deutschland e.V. (VOD)

Deutsche Akademie für Osteopathische Medizin e.V. (DAOM)

Dssociation Luxembourgeoise des Ostéopathes (A.L.D.O.)

ISSN 1610-5044

2003; 1 (3)

Herausgeber :

Prof. Dr. rer. nat. med. habil. Rainer Breul D.O.h.c.

Marina Fuhrmann, D.O. M.R.O.

Prof. Dr. med. habil. Karl-Ludwig Resch

Dr. med. Roger Seider, D.O.

Verlag

Hippokrates Verlag in

MVS Medizinverlage Stuttgart GmbH \& Co.KG

Oswald-Hesse-Str. 50, 70469 Stuttgart, Fax: (07 11) 8931-706 Geschäftsführung:

Dr. med. Thomas Scherb, Dipl. Kaufm. Albrecht Hauff

Programmplanung:

Marina Horbatsch

Fon/Fax: (07 11) 89 31-715/-705

E-Mail: marina.horbatsch@medizinverlage.de und

redaktion.do@medizinverlage.de

Redaktion:

Christoph Newiger in team $4 \mathrm{U}$ Medienbüro

Fon/Fax: (089) 209001-67/-68

E-Mail: redaktion.do@medizinverlage.de

Beratung:

Dorothee Seiz

in MVS Medizinverlage Stuttgart GmbH \& Co. KC

E-Mail: redaktion.do@medizinverlage.de

Marketing:

Marion Krubasik

Fon/Fax: (07 11) 89 31-735/-706

E-Mail: marion.krubasik@medizinverlage.de

Anzeigen:

Günter Fecke, MVS Media-Service

Fon/Fax: (07 11) 8931-714/-706

E Mail: guenter.fecke@medizinverlage.de

Abonnenten-Service

Fon/Fax: (07 11) 8931-321/-422

E Mail: kundenservice@thieme.de

Produktion

Satz und Gestaltung: Fotosatz Sauter, Donzdorf

Druck: Rondo Druck, Ebersbach

Autorenhinweise

Auf Anfrage bei der Redaktion.

Urheberrecht:

Mit der Annahme eines Manuskriptes erwirbt der Verlag für die Dauer der gesetzlichen Schutzfrist ( $\$ 64$ UrhRG) die ausschließliche Befugnis zur Wahrnehmung der Verwertungsrechte im Sinne der $\S \S 15 \mathrm{ff}$. des Urheberrechtsgesetzes. Die Zeitschrift und alle in ihr enthaltenen einzelnen Beiträge und Abbildungen sind für die Dauer des Urheberrechts geschützt. Jede Verwertung ist ohne Zustimmung des Verlages außerhalb der engen Grenzen des UrhRG unzulässig und strafbar. Dies gilt insbesondere für Vervielfältigungen, Übersetzungen, Microverfilmungen und die Einspeicherung und Verarbeitung in elektronischen Systemen. Für den persönlichen Gebrauch dürfen von Beiträgen oder Teilen von diesen einzelne Kopien hergestellt werden. Die Rechte an den Abbildungen liegen

- wenn nicht anders gekennzeichnet - beim Verlag.

Erscheinungsweise

Vierteljährlich

\section{An unsere Leser}

Die Versandkosten für unsere Zeitschrift sind gestiegen. Wir bitten daher um Verständnis, wenn wir uns gezwungen sehen, den Bezugspreis für die „DO Deutsche Zeitschrift für Osteopathie“ ab Januar 2004 neu festzusetzen: Das Jahresabonne-

ment mit $€ 64,90$, Aus-, Fort- und Weiterbildung

$€ 39,90$ zzgl. Versandkosten Inland $€ 6,50$, Europa

$€ 11,20$, Restl. Welt $€ 19,90$.

Einzelheft $€ 18,00$ zzgl. Versandkosten ab Verlagsort. Alle Preise sind unverbindlich empfohlene Preise. Alle Preise und Versandspesen enthalten 7\% MwSt.

\section{Gründung der WOHO - neue Epoche oder „Bullshit“?}

W ährend der Convocation der AAO in Kanada im März 2003 stellte die im September 2002 in Portugal entstandene Taskforce die Gründung der World Osteopathic Health Organisation (WOHO) vor. Ziel der neuen Organisation ist einzig die Förderung der Osteopathie, unabhängig von einzelnen Ländern oder Organisationen. Laut Verfassung soll die WOHO die Philosophie und Praxis der Osteopathie in der gesamten Welt fördern, entwickeln, schützen und etablieren, sowie deren Lehre und Wissen, die Anwendung wie auch die Forschung zum Nutzen der Menschen in der ganzen Welt.

Die gemeinnützige Organisation soll international agieren, der rechtliche Sitz wird jedoch in England und Wales liegen, da hier sowohl osteopathische Ärzte wie auch nichtärztliche Osteopathen rechtlich anerkannt sind. Mitglieder können Einzelpersonen werden, Organisationen sollen dagegen nur als nicht stimmberechtigte unterstützende Mitglieder aktiv werden.

Eigenartig erscheint die Unterscheidung zwischen „Full Registered Members“ und „Full Members“. Im Fall der „Full Registered Members“ muss die Osteopathie in dem Land der Ausübung gesetzlich anerkannt sein, während die „Full Members“ nachweisen müssen, dass sich die Osteopathie in ihrem Land aktiv um eine Anerkennung bemüht. In Deutschland dürfte die zweite Variante in Frage kommen. Durch diese Unterscheidung will man versuchen, auf die Anerkennungsverfahren in den Ländern Druck auszuüben, in denen die Legalisierung noch nicht verwirklicht ist.

Das geplante Konzept hat lebhafte Diskussionen hervorgerufen. Gibt es für einzelne Osteopathen überhaupt einen Anreiz, dieser Organisation beizutreten? Werden die Mehrheitsverhältnisse zwischen den einzelnen Ländern sowie zwischen Osteopathen und osteopathischen Ärzten nicht sehr dem Zufall unterworfen sein, je nachdem, wie es jeweils vor Ort gelingt, Mitglieder zu mobilisieren? Werden bei wechselnden Veranstaltungsorten nicht die Mitglieder des ausrichtenden Landes überrepräsentiert sein? Wer wird schon von Europa aus für ein kurzes Treffen nach Australien fliegen? Insbesondere Dr. Pszolla von der DGOM bezeichnete dieses Konzept schlicht als „Bullshit“. In einer Organisation von Organisationen hätten diese bereits Ressourcen, die in die WOHO eingebracht werden könnten. Positionen wären bei einem überschaubarerem Teilnehmerkreis berechenbarer. Die Taskforce wollte von dieser ursprünglich in Portugal angedachten Form jedoch bewusst Abstand nehmen, da in der Vergangenheit nicht auszumachen war, welche Organisationen in der Lage gewesen wären, ihre Länder alleine und angemessen zu vertreten. Prof. Graf-Baumann (DGOM) kritisiert auch die rechtliche Form der Organisation nach dem „Common Law“ in England und Wales.

Ein totgeborenes Kind also, oder vielleicht doch der Beginn eines großen internationalen Durchbruchs? Möglich ist sicher beides. Positiv stimmte der Elan und die Kreativität der Taskforce, die aus Jane Carreiro, Simon Fielding, Yoshiteru Hiratsuka, Michael Mulholland und Renzo Molinari besteht. Diesen fünf gelang es, große Teile des internationalen Forums optimistisch zu stimmen, so dass zumindest bei diesem Treffen eine Aufbruchstimmung aufkam. Wir werden die Entwicklung sorgfältig beobachten.

Die Herausgeber

Titelbild:

E. Blechschmidt, Die pränatalen Organsysteme des Menschen.

Hippokrates 1973; Bearbeitung: Pdesign A. Page, Stuttgart 2002 